\title{
A case of Paraneoplastic Pemphigus Associated with Castleman's Disease
}

\author{
Um caso de Pênfigo Paraneoplástico (PNP) associado à doença de Castleman
}

\author{
Meihua Zeng ${ }^{1}$ \\ Qingtao Kong ${ }^{1}$
}

\author{
Min Zhang ${ }^{2}$ \\ Hong Sang ${ }^{3}$
}

\author{
Wenxing $\mathrm{Hu}^{1}$ \\ Xiulian $\mathrm{Xu}^{4}$
}

\begin{abstract}
We present a case of PNP associated with Castleman's Disease. We have also reviewed the literature and described the characteristics of the two associated diseases. Gene clonal rearrangement was done to help diagnosis. We used, in addition, stereotactic radiosurgery which, as far as we know, has never before been employed to treat PNP associated with Castleman's Disease. This produced a good response, suggesting that it might be a good alternative treatment for PNP associated with Castleman's Disease when it is too difficult to operate.

Keywords: Giant lymph node hyperplasia; Pemphigus; Review

Resumo: Apresentamos um caso de PNP associada à doença de Castleman.Também revisamos a literatura, e referenciamos as características das duas doenças associadas. Um rearranjo genético clonal foi feito para ajudar o diagnóstico. Além disso, usamos a radiocirurgia que até então nunca havia sido utilizada para tratar PNP associada à doença de Castleman. Esta produziu uma boa resposta, sugerindo que pode ser uma boa alternativa para o tratamento de PNP associada com a doença de Castleman quando é muito difícil fazer uma cirugia convencional. Palavras-chave: Hiperplasia do linfonodo gigante, Pênfigo, Revisão
\end{abstract}

\section{INTRODUCTION}

Paraneoplastic Pemphigus (PNP) is an autoimmune bullous disease that presents with painful mucosal ulcerations and polymorphous skin lesions. Clinically, it is a blistering and erosive mucocutaneous disease associated with an underlying neoplasm. Histologically, it is acantholysis with interface dermatitis or keratinocyte necrosis. Excising the benign neoplasm is the first treatment of choice for the disease, but the CHOP regimen or other, including immunomodulatory, methods are also reported to be useful treatments.

\section{CASE REPORT}

A 49-year-old man presented with oral erosions, erythema and blistering rashes over his trunk and limbs for 3 months. The patient first developed painful oral mucosal blisters and ulcers, which became progressively severe and gradually spread to the eyes, perineum and anus. The patient was admitted to our department for further diagnosis and treatment. On physical examination, we found several small lymph nodes on his neck with diameters of under $1 \mathrm{~cm}$. Dermatologic examination revealed hyperemia on the bilateral conjunctiva, extensive

Received on 03.12.2012

Approved by the Advisory Board and accepted for publication on 20.02.2013

* Work performed at the Dermatology Department, Jinling Hospital, Nanjing University Medical School, China.

Financial Support: None.

Conflict of Interest: None.

MD. Master's degree in medicine. Dermatology Department, Jinling Hospital, Nanjing University Medical School, China.

Attending physician. Master's degree in medicine. Dermatology Department, Jinling Hospital, Nanjing University Medical School, China.

MD and Professor, Dermatology Department, Jinling Hospital, Nanjing University Medical School, China.

MD and Professor, Department of Pathology, Institute of Dermatology, Chinese Academy of Medical Sciences and Peking Union Medical College, Nanjing, China.

(C)2013 by Anais Brasileiros de Dermatologia 
ulcers and erosions on the oral mucosa, erythema and blisters scattered on the chest, back, perineum and anus, but with no obvious ulcers (Figures 1 and 2).

One week prior to admission, the patient had attended another hospital where histological examination, serum direct immunofluorescence (DIF) and indirect immunofluorescence (IIF) were performed. Histological examination of a skin biopsy taken from a lesion on his left arm showed epidermal acantholysis, suprabasal cleft formation, erythrocyte extravasation and vacuolar changes at the dermal epidermal junction and lymphohistiocytic infiltrate in the upper dermis (Figure 3). DIF revealed IgG, IgM, and C3 deposit in the basement membrane zone (BMZ). IIF revealed IgG reticular staining with an antibody titer of 1:20. CT revealed a prominent solid lesion in the right anterior superior mediastinum (Figure 4).

The results of laboratory tests, including full blood count, urea, creatinine, electrolytes, liver function, tumor markers and tests for infectious diseases were normal. Percutaneous mediastinum biopsy guided by CT revealed lymph hematopoietic hyperplasia. Immunohistological examination revealed CD20++, Pax-5++, CD43++, CD3+, CD163+, CD68 scattered+, Mum-1-, CD30-, CD10-, EMA-, CKpan-, TdT-, Ki673\%+, CD5 scattered +.

PCR analysis of Ig heavy chain genes, gene clonal rearrangement (IGH, IGK, IGL, TCR $\beta$, TCR $\gamma$, TCR $\delta$ ) was detected by using BIOMED-2 protocols. DNA extraction from formalin-fixed tissues was performed using standard methods. All the results were negative (Figures 5 and 6).

Diagnosis: 1. Paraneoplastic Pemphigus 2. Castleman's Disease. Upon hospitalization, the patient was given an intravenous methylprednisolone injection 60mg per day and other supportive therapy. A few days later, the lesions started to dry up and form crusts, except for the oral mucosa. According to the advice from the Cardiothoracic Surgery Department, the lesion in the right anterior superior mediastinum was too large to be completely resected, since abundant blood vessels were present and it was too close to the pulmonary artery. In any case, since the patient rejected surgery, conservative treatment was recommended. Considering that the erythema and crusting on the patient's chest and back could perhaps further aggravate after radiotherapy, the Radiotherapy Department attempted to use stereotactic radiosurgery, with an irradiated DT:32Gy/2fx and isodose line $80 \%$. After the end of treatment, the patient's lesions aggravated and new erythema and blisters appeared. However, these recovered during the following week, and no new lesions manifested during the short follow-up period.

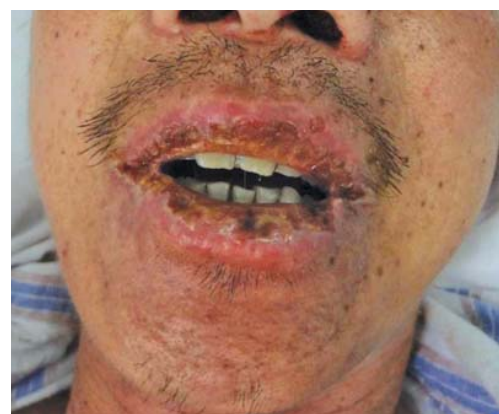

FIGURE 1: Extensive ulcers and erosions on oral mucosa

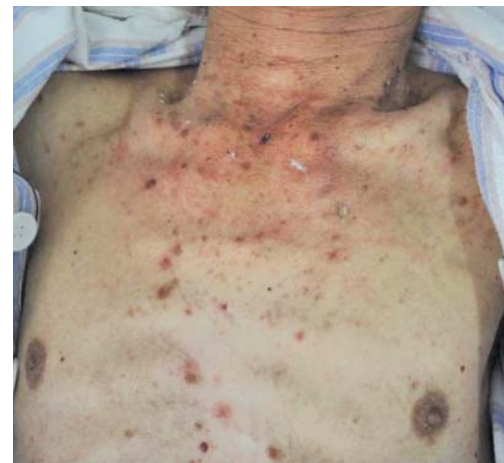

FIGURE 2: Erythema and blisters scattered on the chest

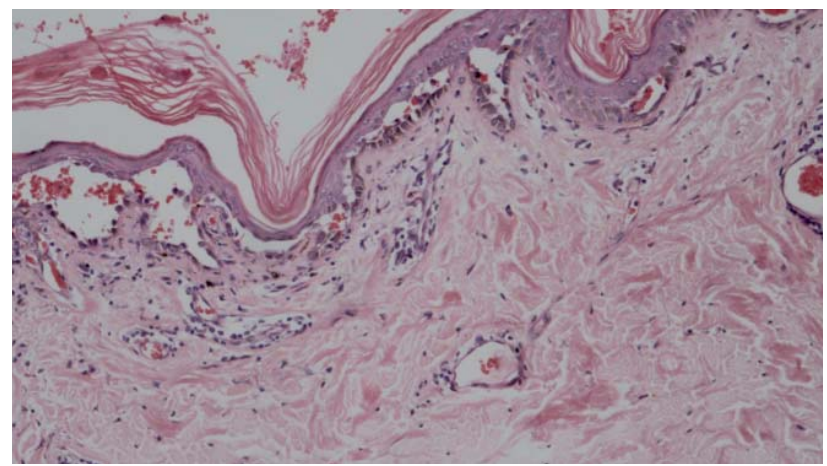

FIGURE 3: $(10 \times 10)$ Histological examination of a skin biopsy taken from a lesion on the patient's left arm revealed epidermal acantholysis, suprabasal cleft formation, hemorrhage in the vacuolar changes at the dermal-epidermal junction. Basal cells on the blister bottom showed a "tombstone" arrangement, lymphohistiocytic infiltrate around the capillaries in the upper dermis, with varying amounts of eosinophils and neutrophils scattered in it

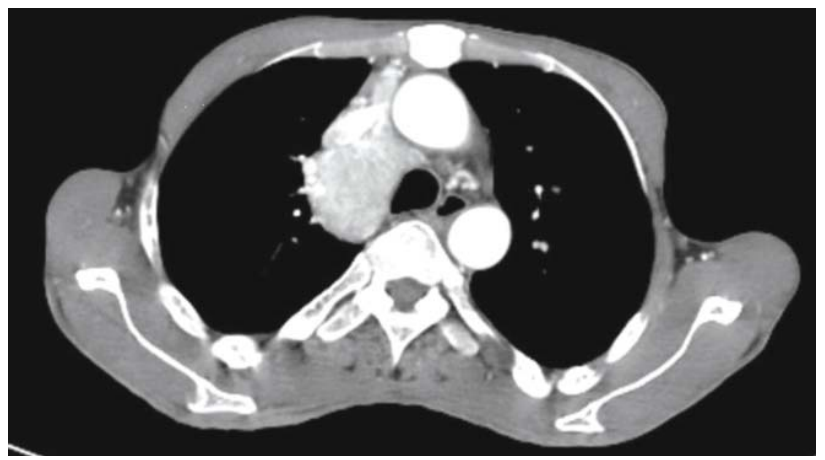

FIGURE 4: CT revealed a prominent solid lesion in the right anterior superior mediastinum, with a clear border, local patch shadow in the upper lobe of the right lung, compressing the trache into the medial deviation and thicker in the cardia wall of the stomach 


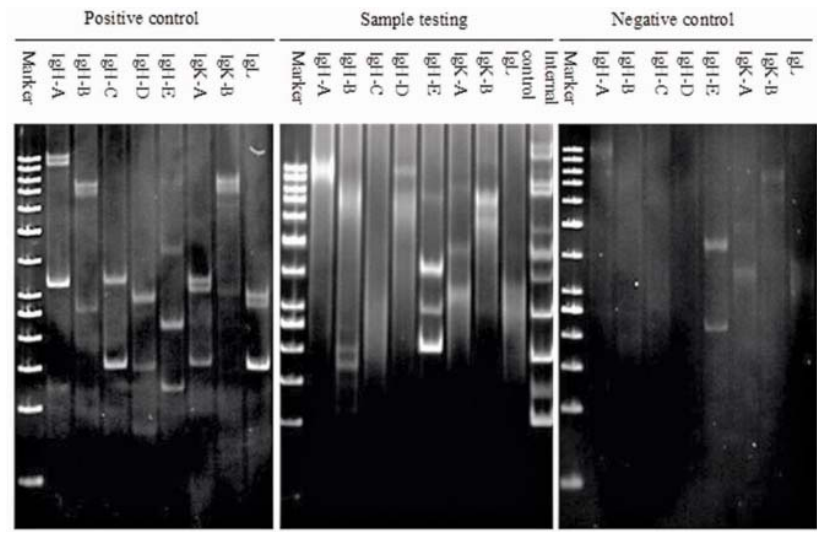

FIGURE 5: PCR analysis of Ig heavy chain genes was negative

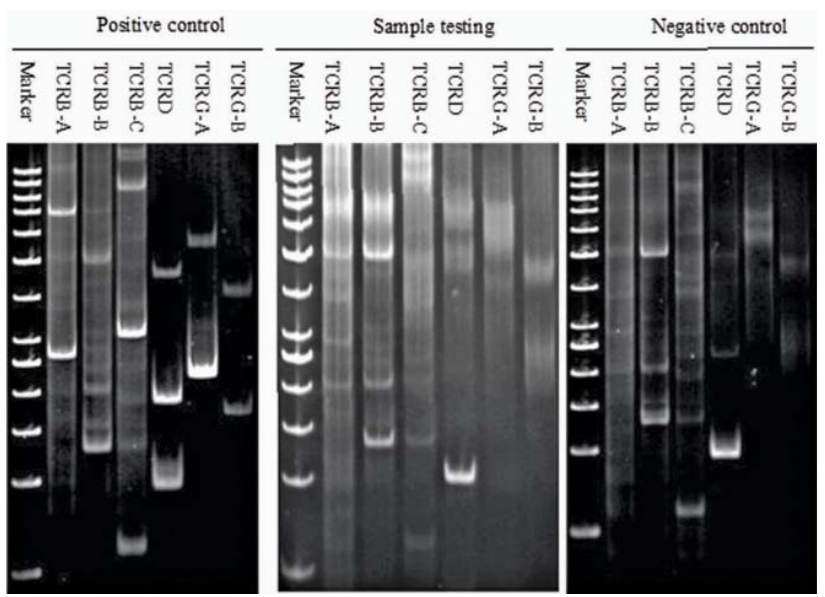

FIGURE 6: Gene clonal rearrangement (IGH, IGK, IGL, TCR $\beta$, TCRY, TCRס) was negative

\section{DISCUSSION}

Paraneoplastic pemphigus (PNP) was first described by Anhalt et al in 1990. ${ }^{1}$ It is associated with non-Hodgkin's lymphoma (42\%), chronic lymphocytic leukemia (29\%), Castleman's disease (10\%), timoma $(6 \%)$, Waldestrom's macroglobulinemia $(6 \%)$, and sarcoma $(6 \%) .^{2}$ Our patient's PNP was associated with Castleman's disease. Clinical classification of focal Castleman's disease or histopathological variants of hyaline vascular are most often associated with the development of paraneoplastic pemphigus, both of clinical and histopathological variants as in the case described. ${ }^{3}$ In 2005, Wang et al presented ten cases of paraneoplastic pemphigus associated with Castleman's disease which were treated in their department. 18 further more cases were reviewed at that time ${ }^{3}$ and since then we discovered that 7 more reported cases. ${ }^{2,-9}$ The clinical manifestation of the disease is variform and may involve mucosa and the skin. According to the data we researched, the mostly common involved mucosal lesions are oral $(97.2 \%)$, followed by anogenital $(72.2 \%)$ and ocular lesions $(58.3 \%)$. The lesions on the mucosa are blisters, erosions, or ulcers, as well as erosive lichen planus-like, or more commonly Stevens-Johnson-like, lesions. Some cases reported only with oral ulcerations. ${ }^{5} \mathrm{We}$ found that, clinically, lichenoid lesions were the most common skin lesions (44.4\%) reported, as well as some atypical manifestations such as flaccid bullae, erythema multiforme-like lesions, and even rash-free cases. ${ }^{3}$ Our patient presented with oral erosions, erythema and blistering rashes over his trunk and limbs which clinically mimicked Stevens-Johnson syndrome.

Excising the benign neoplasm is the first treatment choice for the disease. Seven of the 10 patients treated in Wang's department responded well to general dermatologic management. Both cutaneous lesions and mucosal lesions improved gradually after total resection of the tumors. Wang et al also mentioned in their paper that Gili et al and Jansen et al had reviewed 5 and 12 cases of PNP associated with Castleman's tumor respectively. The lesions in all Gili's patients improved after surgical removal of the tumors, and 8 out of the 12 patients treated by Jansen et al also markedly improved. Other successful reported cases using other treatment methods have also been reported, such as the CHOP regimen and immunomodulation therapy., Neuhof et al analyzed the clinical results of 5 patients with unicentric Castleman's disease treated successfully with radiotherapy. ${ }^{10}$ However, no case was reported that had been treated with stereotactic radiosurgery. In our particular case, stereotactic radiosurgery, with an irradiated DT:32Gy/2fx and isodose line $80 \%$, was considered worth trying. As far as we know this has never been used before, and it produced good responses. It is possible that stereotactic radiosurgery, aimed at controling Castleman's disease, could therefore be a further option for treating Paraneoplastic Pemphigus associated with Castleman's Disease rather than resorting to other, more complex, surgical procedures. 


\section{REFERENCES}

1. Anhalt GJ, Kim SC, Stanley JR, Korman NJ, Jabs DA, Kory M, et al. Paraneoplastic pemphigus: an autoimmune mucocutaneous disease associated with neoplasia. $\mathrm{N}$ Engl J Med. 1990;323:1729-35.

2. Alarcón-Torres I, Bastida-Iñarrea J, Rodríguez-Salido MJ, Gómez-Duaso J, RuaFigueroa I, García-Aguilar GD, et al. Paraneoplastic pemphigus associated with Castleman's disease: usefulness of the laboratory of autoimmunity in the diagnosis of this disease. Ann N Y Acad Sci. 2007;1107:231-8.

3. Wang J, Zhu X, Li R, Tu P, Wang R, Zhang L, et al. Paraneoplastic pemphigus associated with Castleman tumor: a commonly reported subtype of paraneoplastic pemphigus in China. Arch Dermatol. 2005;141:1285-93.

4. Shi BB, Li HZ, Zhao L, Sun Q, Fan H, Li HJ, et al. Paraneoplastic pemphigus caused by Castleman's disease masquerading as an adrenal neoplasm. J Clin Endocrinol Metab. 2009;94:1841-2.

5. Miltenyi Z, Toth J, Gonda A, Tar I, Remenyik E, Illes A. Successful Immunomodulatory Therapy in Castleman's Disease with Paraneoplastic Pemphigus Vulgaris. Pathol Oncol Res. 2009;15:375-81.

6. Tey HL, Tang MB. A case of paraneoplastic pemphigus associated with Castleman's disease presenting as erosive lichen planus. Clin Exp Dermatol. 2009;34:e754-6.

7. Singh M, Saroha V, Gupta P, Gupta P, Khurana N, Singh T. Hyaline vascular Castleman's disease relapsing as T cell rich B cell lymphoma with paraneoplastic pemphigus. J Clin Pathol. 2011;64:93-4.

8. Jindal T, Meena M, Kumar A, Khaitan BK. Paraneoplastic pemphigus with Castleman's disease and bronchiolitis obliterans. Pediatr Int. 2011;53:1108-9.

9. Wen X, Jiang X. Paraneoplastic pemphigus in association with Castleman's disease of the pararenal retroperitoneum. J Dermatol. 2012;39:662-4.

10. Neuhof D, Debus J. Outcome and late complications of radiotherapy in patients with unicentric Castleman's disease. Acta Oncol. 2006;45:1126-31.
MAILING ADDRESS:

Hong Sang

$N^{\circ} .305$ - Zhongshan East Road - Nanjing City

210002 - Jiangsu - China

E-mail: sanghong@nju.edu.cn

Xiulian Xu

No.12 - Jiangwangmiao Road - Nanjing City

210042 - Jiangsu - China

E-mail:xxlqjl@sina.com

How to cite this article: Zeng M, Zhang M, Hu W, Kong Q, Sang H, Xu X. A case of Paraneoplastic Pemphigus associated with Castleman's Disease. An Bras Dermatol. 2013;88(6 Suppl 1):S11-4. 